\title{
Volumetric Raman chemical imaging of drug delivery systems
}

Slipets, Roman; Ilchenko, Oleksii; Mazzoni, Chiara; Tentor, Fabio; Nielsen, Line Hagner; Boisen, Anja

Published in:

Journal of Raman Spectroscopy

Link to article, DOI:

$10.1002 /$ jrs.5869

Publication date:

2020

Document Version

Peer reviewed version

Link back to DTU Orbit

Citation (APA):

Slipets, R., Ilchenko, O., Mazzoni, C., Tentor, F., Nielsen, L. H., \& Boisen, A. (2020). Volumetric Raman chemical imaging of drug delivery systems. Journal of Raman Spectroscopy, 51(7), 1153-1159.

https://doi.org/10.1002/jrs.5869

\section{General rights}

Copyright and moral rights for the publications made accessible in the public portal are retained by the authors and/or other copyright owners and it is a condition of accessing publications that users recognise and abide by the legal requirements associated with these rights.

- Users may download and print one copy of any publication from the public portal for the purpose of private study or research.

- You may not further distribute the material or use it for any profit-making activity or commercial gain

- You may freely distribute the URL identifying the publication in the public portal

If you believe that this document breaches copyright please contact us providing details, and we will remove access to the work immediately and investigate your claim 


\title{
Volumetric Raman chemical imaging of drug delivery systems
}

\author{
Roman Slipets, Oleksii Ilchenko*, Chiara Mazzoni, Fabio Tentor, Line Hagner Nielsen, Anja Boisen
}

The Danish National Research Foundation and Villum Foundation's Center for Intelligent Drug Delivery and Sensing Using Microcontainers and Nanomechanics (IDUN) - Department of Health Technology, Technical University of Denmark, Kgs. Lyngby, Denmark

\begin{abstract}
The ability to image drug distribution inside a several hundred-micron thick polymer matrix, encapsulated by a protective coating, would greatly contribute to the understanding of the performance and shortcomings of drug delivery devices. Here, we present an experimental framework for deep volumetric Raman imaging (dVRI), where common challenges such as low Raman cross section, fluorescence and low transparency of samples are overcome. We apply dVRI to a selection of drug delivery forms; tablets with thin protective coatings and drug-loaded microdevices. We demonstrate 3D visualization of the different drug/polymeric materials, constituting a drug delivery device, with imaging depth of $225 \mu \mathrm{m}$.
\end{abstract}

Key words: volumetric mapping, Raman imaging, decomposition, chemometrics, drug delivery systems

\section{INTRODUCTION}

Three-dimensional (3D) confocal Raman mapping is one of the most promising techniques to study the chemical composition of complex organic ${ }^{[1]}$ and inorganic materials ${ }^{[2]}$. The common way to present Raman data is based on peak intensity visualization. However, this method leads to artifacts in the visualization of chemical responses due to overlapping of the spectral profiles from different compounds ${ }^{[3]}$. In order to overcome this problem, several chemometric approaches have been developed ${ }^{[4-8]}$. It has recently been demonstrated that quantitative volumetric Raman imaging (qVRI) can be applied to cell culture samples ${ }^{[1]}$ where a Vertex Component Analysis (VCA) algorithm is used ${ }^{[9]}$. However, qVRI is still a state-of-art technique with few reported applications, mostly due to interference from molecular fluorescence ${ }^{[10]}$, limited sample transparency at the laser excitation wavelength ${ }^{[11,12]}$, complexity of chemometric analysis ${ }^{[13]}$ and low Raman scattering cross section of the chemical components ${ }^{[4]}$. Herein, we demonstrate that these obstacles can be overcome, using tablets with thin protective coatings and drug-loaded polymeric microcontainers (MCs) as challenging test cases. MCs are cylindrical microdevices with only the top side open, fabricated in the epoxy polymer SU-8 $8^{[14]}$. It has been shown that MCs improve oral bioavailability of model drugs due to their unidirectional release ${ }^{[15,16]}$. Our studied MCs are loaded with a polymer matrix (polyvinylpyrrolidone, PVP) and a drug (i.e. ketoprofen, naproxen) and used for oral drug delivery ${ }^{[15]}$. The MC material, SU-8 is highly fluorescent ${ }^{[17]}$ and the microcontainer cavity, loaded with the PVP/drug matrix (low optical transparency), is approximately $225 \mu \mathrm{m}$ deep $^{[15]}$.

The molecular fluorescence of the drug compounds can be suppressed in several ways ${ }^{[18]}$. However, the most proper and common techniques like Kerr-gated Raman ${ }^{[19]}$, UV Raman ${ }^{[20]}$ and near-IR Fourier Transform (FT)-Raman ${ }^{[21]}$ are not well applicable to the diffraction limited dVRI due to the limitation connected with confocality or penetration depth. The most appropriate solution is the usage of near IR lasers (780 nm, $785 \mathrm{~nm}, 830 \mathrm{~nm})$ as excitation sources $^{[13]}$. For the sole purpose of suppressing sample fluorescence, Nd:YAG lasers with $1064 \mathrm{~nm}$ excitation wavelength are a very attractive solution ${ }^{[22]}$, however, the Raman scattering cross section becomes usually too low for dVRI. In this situation, where the fluorescence of drug systems takes place, we decided to use NIR laser with wavelength of $785 \mathrm{~nm}$.

Different drug systems have previously been imaged with Raman spectroscopy. However, the transparency is limited, even when using a laser at $785 \mathrm{~nm}$ excitation wavelength ${ }^{[23]}$. Using a commercially available Raman microscope equipped with $785 \mathrm{~nm}$ laser excitation, we were able to scan only the top $15 \mu \mathrm{m}$ of $\mathrm{MCs}^{[24]}$. It is important 
to mention that in-depth Raman mapping is also limited by off-axis laser refraction effects leading to Raman signal attenuation and decreased axial resolution ${ }^{[25-28]}$. The usual way of addressing this problem is to increase the laser power and/or the exposure time. However, the latter leads to an unrealistic total time of 3D map acquisition ( $>3$ days). Increasing the laser power, instead, overheats and burns the sample.

Herein, we present an efficient solution to solve the previously discussed problems of dVRI of samples that manifest fluorescence and low transparency. This result was achieved by the development of a confocal Raman microscope with high Raman signal throughput, optimization of sample mapping method and further chemometric hyperspectral data analysis based on Non-Negative Least Squares (NNLS) ${ }^{[3]}$. Preliminary experimental results were shown in our previous study ${ }^{[29]}$. Here, we present a detailed description of the optical and analytical solutions implemented in the dVRI method.

\section{INSTRUMENTATION}

For dVRI, an optical setup should have diffraction limited confocal performance, high throughput from sample to detector and spectral resolution limited within a spectroscopic sensor pixel size.

To match the required conditions, we designed and constructed a high throughput confocal Raman microscope based on an on-axis lens type spectrograph (Fig. 1a). Design of the optics was performed in Zemax Optics Studio 18.9. As an excitation source, a single mode frequency stabilized laser from Thorlabs LP785-SAV50 (785 nm, 50 $\mathrm{mW}$ ) was used. Lenses $\mathrm{f} 1, \mathrm{f} 2$ were used as a beam expander for the proper fit between the diameter of the laser beam and the microscope objective pupil lens. A Zeiss objective 100x/0.75 HD DIC was used.

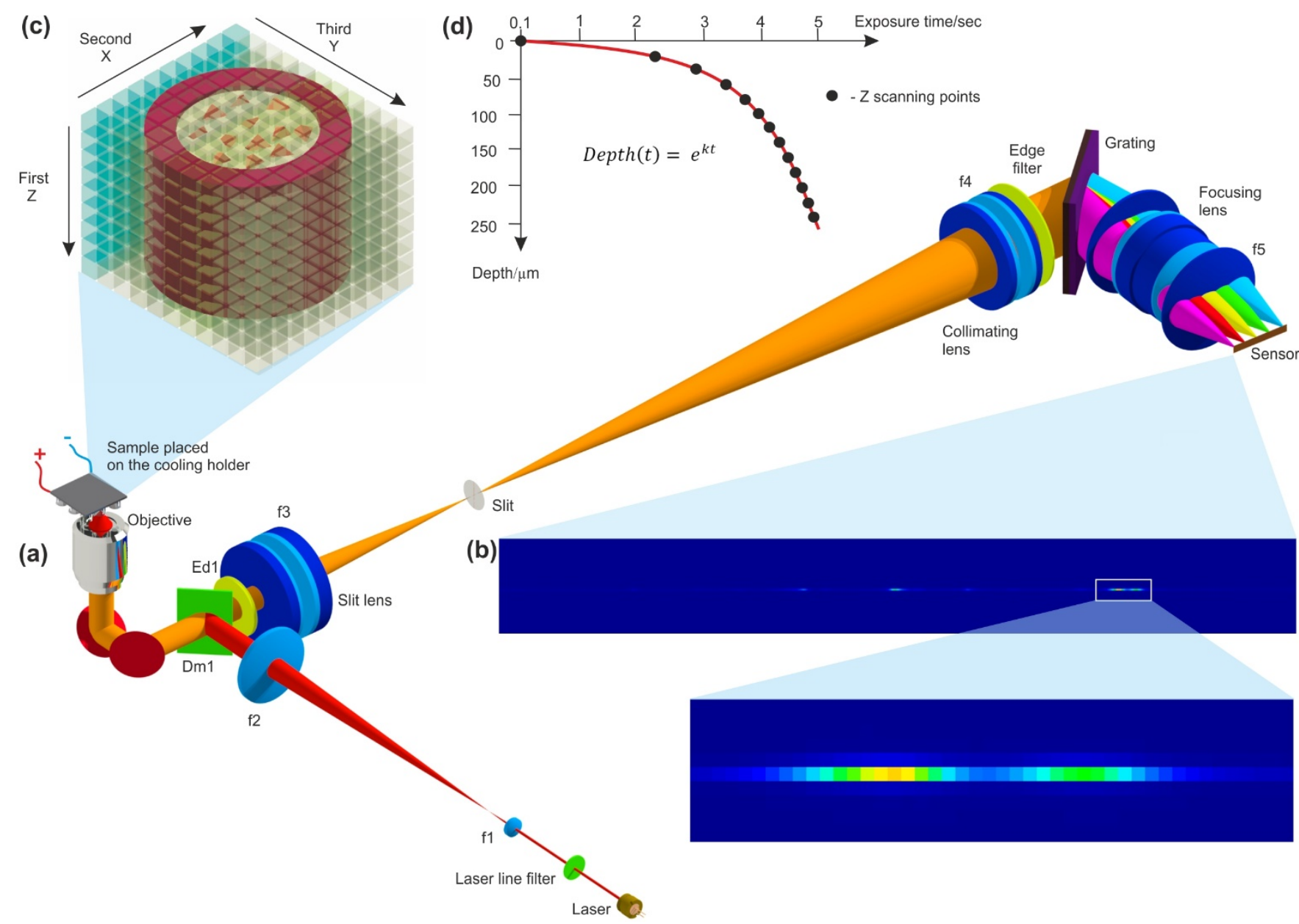

Figure 1. Deep and highly sensitive volumetric Raman mapping (dVRi). (a) Optical design of the Raman microscope, (b) example of an image from the spectroscopic CCD Raman signal; the zoomed region shows that the spectrum is compressed into one row on the CCD sensor, (c) $Z \rightarrow X \rightarrow Y$ mapping algorithm applied to the MC measurements for a more homogeneous heat distribution, (d) depth dependent acquisition algorithm which exponentially increases the exposure time for each mapping z-stack. 
A detailed description of the microscope's X,Y,Z scanning procedure has been reported earlier ${ }^{[13]}$.

The collimated Raman beam from the microscope objective is propagated through the dichroic mirror Dm1 and the edge filter Ed1 and focused on the spectrograph slit by the lens f3. The spectrograph consists of fused silica transmitting grating (averaged diffraction efficiency $96 \%$ ), collimating lens $\mathrm{f} 4$ and focusing lenses f5 (see details in ${ }^{[13]}$ ). As an imaging sensor, we used a near IR enhanced deep cooling CCD (Andor iDus416).

Due to the aberration corrected Raman system design, diffraction limited axial resolution was reached without a classical pinhole ${ }^{[13]}$. The confocal mode was organized in a cross slit geometry in which, the vertical slit orientation was implemented in the entrance of the spectrometer and, the horizontal slit orientation was organized on the spectroscopic CCD focal plane by the readout of the selected rows. An example of a typical Raman spectrum measured with our system is shown in Fig. 1b. In the zoomed region, it is possible to observe that the Raman spectrum is compressed into one row on a spectroscopic sensor. Due to the absence of moving parts, extra mirrors and the usage of custom aberration corrected optics with NIR coatings, high total Raman system throughput (from sample to detector) was reached at the level of $91 \%{ }^{[30]}$.

\section{METHODS}

To address the problem of the Raman signal attenuation taking place in tablets and MCs, we designed and constructed a highly sensitive confocal Raman microscope (Fig. 1a). However, even in this case, we verified that the Raman signal attenuation at an MC depth of $100 \mu \mathrm{m}$ was significant and, consequently, the system was insufficient for mapping the total depth of the MC $(225 \mu \mathrm{m})$. As a solution, we decided to increase the laser power delivered to the sample from $5 \mathrm{~mW}$ to $30 \mathrm{~mW}$. To efficiently remove the heat generated by the laser, a Peltier cooling system of the sample holder was implemented. The temperature was kept at $8^{\circ} \mathrm{C}$ during all $3 \mathrm{D}$ mapping experiments. The 3D mapping was organized so that the first mapped axis dimension was " $Z$ ", the second - " $X$ " and the third - "Y" (Fig. 1c). This approach provided a more homogeneous heat distribution through the volume of tablets and MCs in comparison to a traditional mapping where the laser is kept at one $\mathrm{Z}$ stack for much longer time.

In order to maintain the signal-to-noise (SNR) ratio of Raman spectra captured at different depths in the same value range, we performed a depth dependent acquisition algorithm which exponentially increases the exposure time for each Z stack mapped (Fig. 1d).

All of the presented volumetric Raman maps were measured at the following conditions: the exposure time per point varied from $0.1 \mathrm{~s}$ to $3 \mathrm{~s}$ (exponentially scaled depending on the mapping depth), the CCD was operated in a single track mode with vertical beaning equal to 4 pixels. The step size was 6 and $12 \mu \mathrm{m}$ in lateral and axial dimensions, respectively, 3D map dimensions were 50x50x25 pixels or 300x300x300 $\mu \mathrm{m}$. The total measurement time per map was equal to $26 \mathrm{~h}$. All spectra were background corrected using rolling-circle filter ${ }^{[31]}$ (circle radius was $\left.300 \mathrm{~cm}^{-1}\right)$. Savitzky-Golay filtering algorithm was applied for data smoothing ${ }^{[32]}$. All acquisition and data analysis procedures including Non-Negative Least Squares (NNLS) were realized in our own software written in Delphi. 


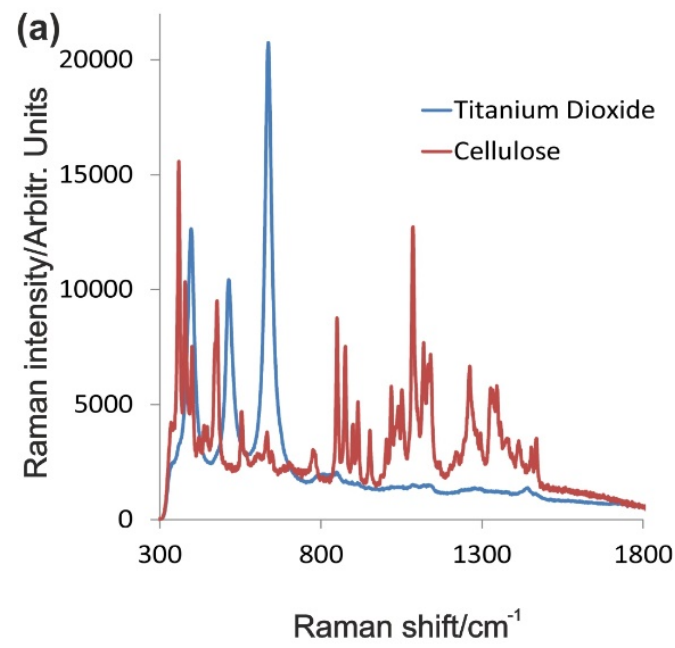

(c)

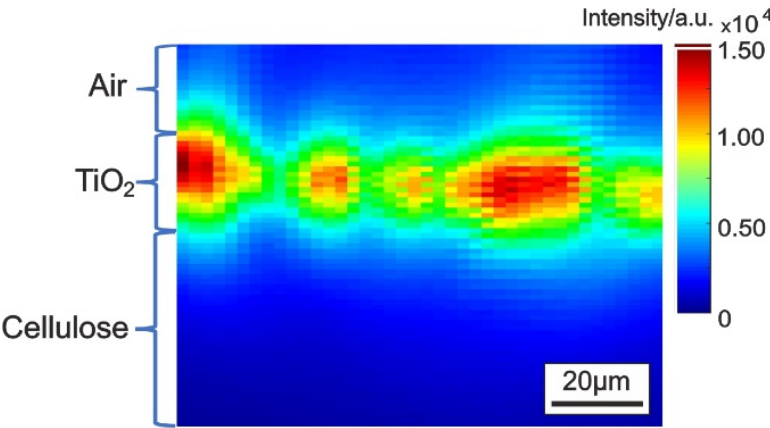

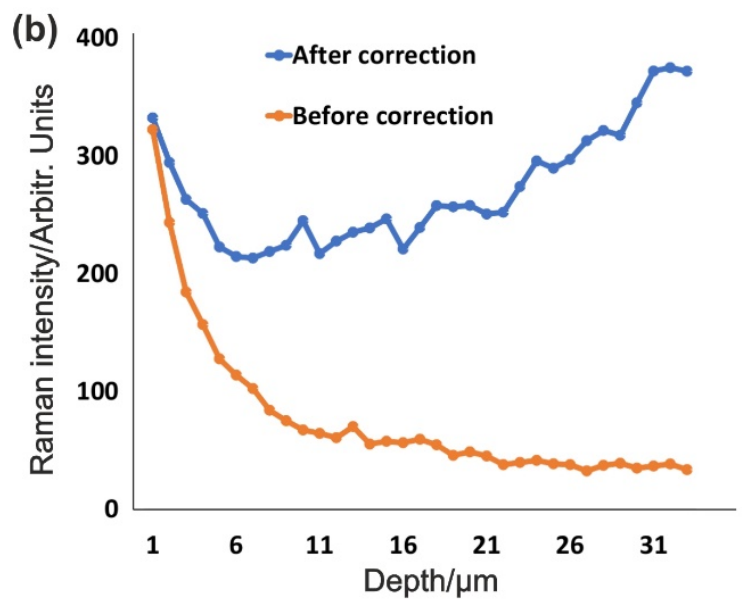

(d)

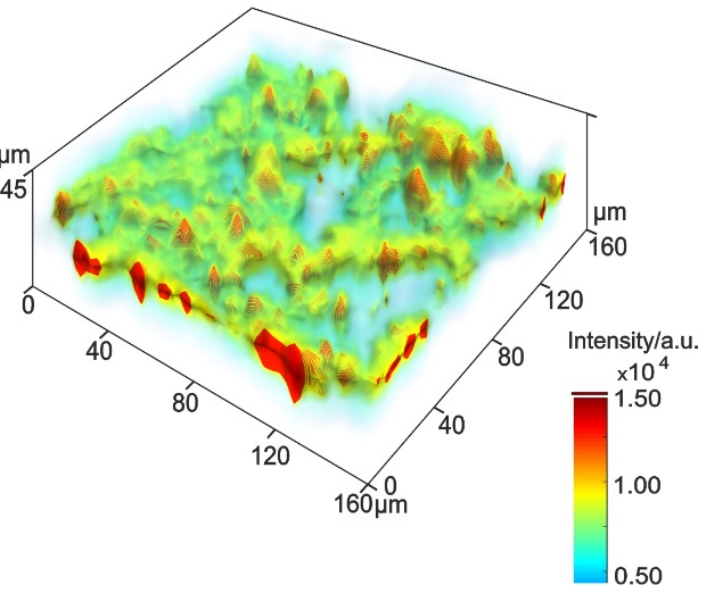

Figure 2. dVRi of a pharmaceutical tablet. (a) Raman spectra of a model tablet consisting of titanium dioxide coating and cellulose, (b) depth dependent Raman intensity profile of the tablet matrix (cellulose) before (orange line) and after (blue line) exponential correction, (c) depth cross section of the cellulose tablet coated with titanium dioxide, (d) dVRI map of titanium dioxide coating represented at peak intensity of Eg mode $\left(635 \mathrm{~cm}^{-1}\right)$ with an imaging depth of $45 \mu \mathrm{m}$.

\section{RESULTS}

As a demonstration example of the performance of dVRI, we used a pharmaceutical tablet consisting of a cellulose matrix (Fig. 2). The depth dependent exponential decay of the intensity of the Raman spectrum of cellulose (Fig. $2 \mathrm{a}$ (red line)) is shown in Fig. $2 \mathrm{~b}$ (orange line). The Raman signal intensity after exponential exposure time correction is shown in Fig. $2 b$ (blue line). In a homogenous medium, the presented dependence should have a constant Raman intensity versus tablet depth. The deviation from constant dependence behavior was caused by inhomogeneity of material compression at the stage of tablet production ${ }^{[33]}$. Nevertheless, we demonstrated that applied correction can significantly improve SNR ratio in depth dependent Raman mapping. The depth cross section and the dVRI map of titanium dioxide coating on the surface of the cellulose tablet represented at peak intensity of Eg mode $\left(635 \mathrm{~cm}^{-1}\right)$ and is shown on Fig. $2 \mathrm{c}, 2 \mathrm{~d}$ (Movie S1). It is possible to observe small titanium dioxide clusters on the surface of the tablet. This result demonstrates that dVRi is applicable for coating thickness determination, as well as for verification of coating homogeneity. Such information is crucial and can be used to optimize a tablet manufacturing process.

As an example of dVRi applicability for multicomponent polymeric devices for drug delivery, we demonstrate results obtained on MCs. Ketoprofen and naproxen are poorly soluble drugs and it was of utmost important to evaluate their solid state once loaded into MCs. The solid state influences the dissolution of the drugs. For the purpose of chemical decomposition, we measured Raman spectra of the pure chemical components presented in the MCs (Fig. S1a). Following this, we identified the peak positions of amorphous and crystalline drug (ketoprofen), PVP, SU-8 and Si with minimum peak overlapping. The selected peaks are $1000 \mathrm{~cm}^{-1}, 1688 \mathrm{~cm}^{-1}, 932.5 \mathrm{~cm}^{-1}, 809$ $\mathrm{cm}^{-1}$ and $520 \mathrm{~cm}^{-1}$, respectively. 
The Raman intensity-based 3D plots are shown in Fig. S1b. We do not present a map of crystalline ketoprofen as the response of crystalline drug was not observed in the Raman map. The problem of overlapping Raman peaks prevents the use of univariate data-analysis to map the distribution of components: ketoprofen, PVP and Si responses are all present on the walls of the MCs (Fig. S1a, S1b). Drawing conclusions on the drug distribution is thereby not possible. To solve this issue a chemometric hyperspectral data analysis was applied.

Species contributions in multicomponent mixtures can be determined from the spectral data using different chemometric techniques ${ }^{[4,8,9]}$. Most algorithms are based on the fact that the recorded spectra are the weighted sum of pure components spectra present in the investigated mixture. Based on this assumption, the unfolded data matrix $\mathbf{X}$ may be decomposed into a concentration matrix $\mathbf{C}$ and a spectral matrix $\mathbf{S}^{\mathrm{T}}$

$$
\mathbf{X}=\mathbf{C S}^{\mathbf{T}}+\mathbf{E}
$$

where $\mathbf{E}$ is the residual matrix (Fig. 3a).

When the pure spectrum of each component is known, their concentration can be obtained directly using a classical least squares (CLS) $\operatorname{approach}^{[3]}$ :

$$
\mathbf{C}=\mathbf{D S}\left(\mathbf{S}^{\mathrm{T}} \mathbf{S}\right)^{-1}
$$

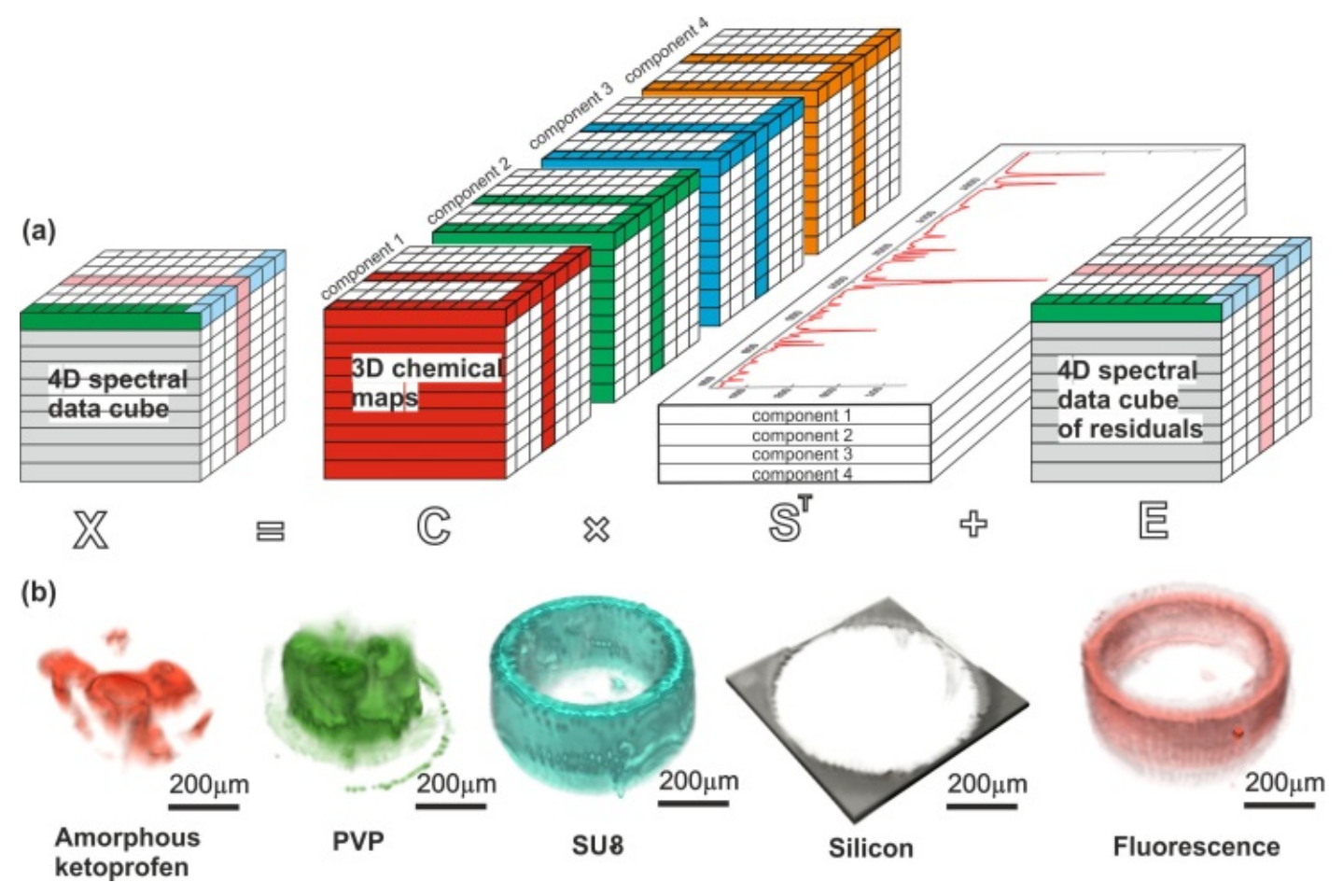

Figure 3. Volumetric chemical imaging. (a) Illustration of the NNLS algorithm for the decomposition of hyperspectral Raman data into normalized chemical responses, (b) volumetric chemical maps of MCs components (amorphous ketoprofen, PVP, SU-8, Si) built after NNLS analysis. The volumetric distribution of fluorescence is shown to the right.

The limitation of CLS lies in the assumption that the separately measured pure spectra of the chemical components $\mathbf{S}^{\mathrm{T}}$ have identical profiles in the mixture (experimentally measured matrix $\left.\mathbf{D}\right)^{[3]}$. In the case of the MCs analysis, we were able to (i) measure the pure spectra of each component (Fig. S1a) and (ii) demonstrate that there were no chemical interactions occurring between components in the loaded MCs. Therefore, CLS algorithm was a perfect solution for our case study. The LS was limited with two constrains, applied to the matrix $\mathbf{C}$ : non-negativity and 
normalization. Such algorithm is usually called Non-Negative Least Square (NNLS) analysis ${ }^{[3]}$. As an extra component, in $\mathbf{S}^{\mathrm{T}}$ we also included a spectrum generated from the lenses of the microscope objective. Finally, we obtained 3D plots of the normalized distributions of amorphous ketoprofen, PVP, SU-8 and Si (Fig. 3b). Microscope objective response was excluded from the normalization procedure since it is not involved in the set of chemical components of MCs. Comparing with the 3D maps obtained by the peak intensity (Fig. S1b), NNLS decomposed maps of MCs components were clearly separated and ready for distribution analysis.

Since the acquired spectral data set (matrix $\mathbf{X}$ ) was background corrected, the presence of fluorescence was subtracted from the impact into the decomposition result. However, the residuals obtained after the background subtraction were used for the representation of the distribution of fluorescence response (see pink image on Fig. 3b). It mainly represents the fluorescence from SU-8 and fluorescent impurities outside of the MC.

(a)
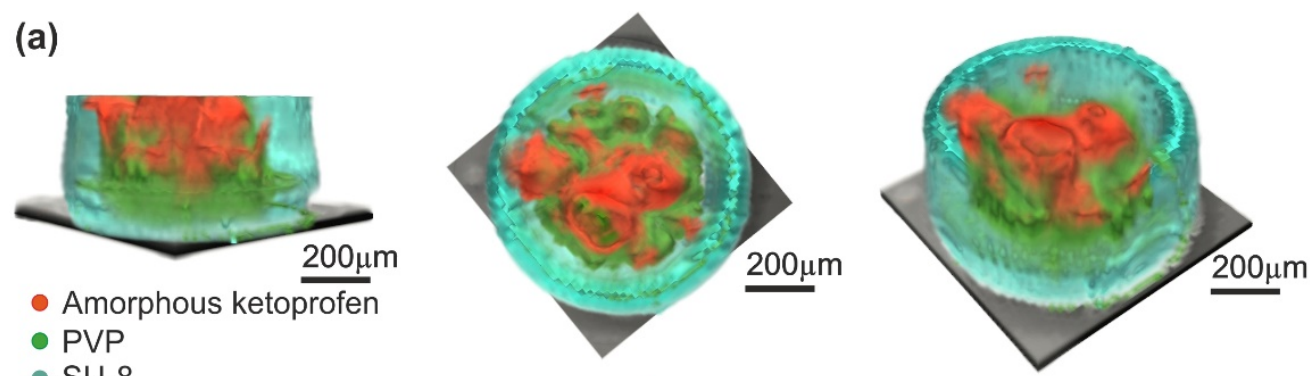

- SU-8

- $\mathrm{Si}$

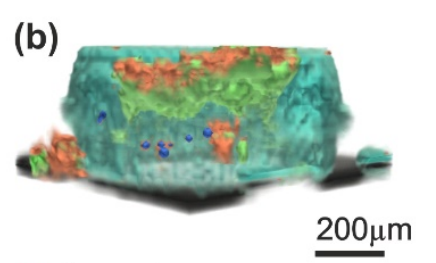

- Amorphous naproxen
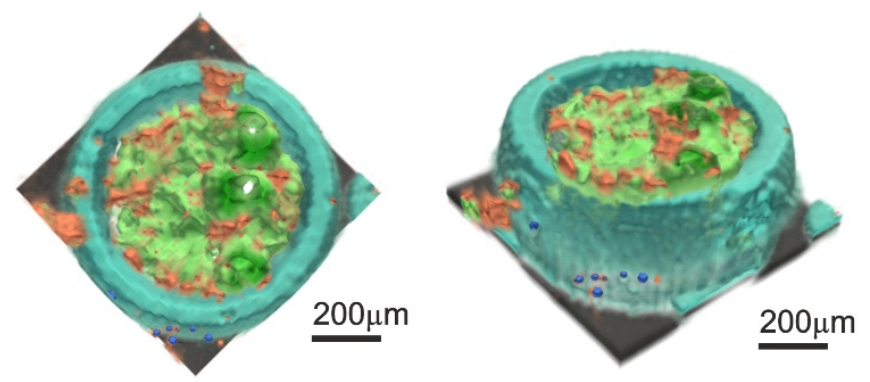

- Crystalline naproxen

- PVP

- SU-8

- $\mathrm{Si}$

Figure 4. Combined volumetric chemical maps of MCs loaded with ketoprofen (a) and naproxen (b) are shown from different rotation angles.

In Fig. 4a, we present the combined volumetric chemical maps of the MCs components. It is possible to conclude that i) PVP was homogeneously distributed throughout the MCs, ii) amorphous ketoprofen was deposited on the top of MCs walls and over the surface of PVP, iii) no signal from crystalline ketoprofen was recorded as we obtained zero values from the crystalline ketoprofen channel, and iv) the Si substrate response was recorded only at the bottom of the map. More visual information can be obtained from supplementary Movie S2.

The universality of the developed method for highly sensitive volumetric Raman chemical imaging of fluorescent and highly absorptive samples was tested on several drugs and MCs with different dimensions in our previous study ${ }^{[2]}$. As an example, the distribution of naproxen loaded into the polymer matrix is shown on Fig. 4b (Movie S3). In this case, naproxen was concentrated on the top of the MCs, with limited penetration into the PVP matrix. A minor amount of crystalline drug was observed outside the MCs (Fig. 4b). It is important to notice that we were not able to distinguish crystalline naproxen in our previous work ${ }^{[29]}$ due to incomplete methodology of dVRi. In particular, fluorescence impact (see Fig. 3b) was not taken into account.

\section{CONCLUSIONS}

A deep and highly sensitive volumetric Raman chemical imaging method has been developed and applied for monitoring the coating thickness on pharmaceutical tablets and the distribution of different components in a drug-loaded 
polymer matrix in MCs for oral drug delivery of poorly soluble drugs. According to the location and the solid state of the drug, it is therefore possible to better understand and predict its release in vitro and/or in vivo. A confocal Raman microscope with improved sensitivity and diffraction limited axial resolution was developed for this purpose. For efficient mapping of the absorptive MCs material, exponential depth dependent exposure correction was applied and the sample was kept under continuous cooling for efficient heat removal. The obtained 3D Raman maps of MCs have been decomposed into chemical volumetric images by the usage of NNLS analysis, where the signal of fluorescence was extracted from background correction as an individual contribution. Crucial information on the distribution/state of drugs in the polymer matrix (PVP) in MCs material (SU-8) was hereby obtained.

\section{AUTHOR INFORMATION}

Corresponding Authors

* E-mail: olil@dtu.dk

\section{Notes}

The authors declare no competing financial interest.

\section{ACKNOWLEDGMENT}

The authors would like to acknowledge the Center for Intelligent Drug Delivery and Sensing Using Microcontainers and Nanomechanics (IDUN) whose research is funded by the Danish National Research Foundation (DNRF122) and Villum Fonden (Grant No. 9301).

\section{REFERENCES}

[1] C. Kallepitis, M. S. Bergholt, M. M. Mazo, V. Leonardo, S. C. Skaalure, S. A. Maynard, M. M. Stevens, , DOI:10.1038/ncomms14843.

[2] A. Zoubir, Ed., Raman Imaging, Springer Berlin Heidelberg, Berlin, Heidelberg, 2012, vol. 168.

[3] O. O. Ilchenko, Y. V. Pilgun, A. S. Reynt, A. M. Kutsyk, Ukr. J. Phys. 2016, 61, 519.

[4] K. C. Gordon, C. M. McGoverin, Int. J. Pharm. 2011, 417, 151.

[5] B. Vajna, G. Patyi, Z. Nagy, A. Bódis, A. Farkas, G. Marosi, J. Raman Spectrosc. 2011, 42, 1977.

[6] Y. Roggo, K. Degardin, P. Margot, Talanta 2010, 81, 988.

[7] J. Jaumot, A. de Juan, R. Tauler, Chemom. Intell. Lab. Syst. 2015, 140, 1.

[8] A. de Juan, R. Tauler, Anal. Chim. Acta 2003, 500, 195.

[9] J. M. P. Nascimento, J. M. B. Dias, IEEE Trans. Geosci. Remote Sens. 2005, 43, 898.

[10] D. Wei, S. Chen, Q. Liu, Appl. Spectrosc. Rev. 2015, 50, 387.

[11] N. J. Everall, Analyst 2010, 135, 2512.

[12] N. J. Everall, Appl. Spectrosc. 2000, 54, 1515.

[13] O. Ilchenko, Y. Pilgun, T. Makhnii, R. Slipets, A. Reynt, A. Kutsyk, D. Slobodianiuk, A. Koliada, D. Krasnenkov, V. Kukharskyy, Vib. Spectrosc. 2016, 83, 180.

[14] J. Nagstrup, S. Keller, K. Almdal, A. Boisen, Microelectron. Eng. 2011, 88, 2342. 
[15] C. Mazzoni, F. Tentor, S. A. Strindberg, L. H. Nielsen, S. S. Keller, T. S. Alstrøm, C. Gundlach, A. Müllertz, P. Marizza, A. Boisen, J. Control. Release 2017, 268, 343.

[16] L. H. Nielsen, A. Melero, S. S. Keller, J. Jacobsen, T. Garrigues, T. Rades, A. Müllertz, A. Boisen, Int. J. Pharm. 2016, 504, 98.

[17] A. Qualtieri, T. Stomeo, L. Martiradonna, R. Cingolani, M. De, in Lithography, InTech, 2010.

[18] A. C. De Luca, M. Mazilu, A. Riches, C. S. Herrington, K. Dholakia, Anal. Chem. 2010, 82, 738.

[19] P. Matousek, M. Towrie, C. Ma, W. M. Kwok, D. Phillips, W. T. Toner, A. W. Parker, J. Raman Spectrosc. 2001, 32, 983 .

[20] S. Asher, C. Johnson, Science (80-. ). 1984, 225, 311.

[21] J. Pironon, J. Sawatzki, D. J., Geochim. Cosmochim. Acta 1991, 55, 3885.

[22] A. P. Oliveira, R. A. Bitar, L. Silveira, R. A. Zângaro, A. A. Martin, Photomed. Laser Surg. 2006, $24,348$.

[23] C. Adlhart, W. Baschong, Int. J. Cosmet. Sci. 2011, 33, 527.

[24] M. Chiara, T. Fabio, A. Anastasia, J. Rasmus, M. Jacob, S. Roman, I. Oleksii, K. S. N. Line, B. Anja, in Distribution and quantitative analyses of poorly water soluble drugs loaded by supercritical CO2 impregnation in microcontainers with different sizes, 2018 CRS Annual Meeting, New York, 2018.

[25] N. J. Everall, Appl. Spectrosc. Vol. 54, Issue 6, pp. 773-782 2000, 54, 773.

[26] J. L. Bruneel, J. C. Lassègues, C. Sourisseau, J. Raman Spectrosc. 2002, 33, 815.

[27] C. Sourisseau, P. Maraval, Appl. Spectrosc. Vol. 57, Issue 11, pp. 1324-1332 2003, 57, 1324.

[28] G. Gouadec, L. Bellot-Gurlet, D. Baron, P. Colomban, 2012, pp. 85-118.

[29] C. Mazzoni, F. Tentor, A. Antalaki, R. D. Jacobsen, J. Mortensen, R. Slipets, O. Ilchenko, S. S. Keller, L. H. Nielsen, A. Boisen, ACS Biomater. Sci. Eng. 2019, 5, 2935.

[30] O. Ilchenko, Nat. Commun., DOI:10.1038/s41467-019-13504-8.

[31] N. N. Brandt, O. O. Brovko, A. Y. Chikishev, O. D. Paraschuk, Appl. Spectrosc. 2006, 60, 288.

[32] M. Člupek, P. Matějka, K. Volka, J. Raman Spectrosc. 2007, 38, 1174.

[33] I. C. Sinka, J. C. Cunningham, A. Zavaliangos, J. Pharm. Sci. 2004, 93, 2040.

\section{Graphical Table of Contents}

Volumetric Raman chemical imaging of drug delivery systems.

Roman Slipets, Oleksii Ilchenko*, Chiara Mazzoni, Fabio Tentor, Line Hagner Nielsen, Anja Boisen

An experimental framework for deep volumetric Raman imaging (dVRI) is presented. Common challenges such as low Raman cross section, fluorescence and low transparency of samples are overcome. We apply dVRI to a selection of drug delivery forms; tablets with thin protective coatings and drug-loaded microdevices and demonstrate $3 \mathrm{D}$ visualization of the different drug/polymeric materials, constituting a drug delivery device, with imaging depth of $225 \mu \mathrm{m}$. 


$$
\text { t: }
$$

